\title{
Students as Partners Pedagogical APPRoACh in EngineEring Education: A CaSe Study ANd Multiple Perspective Reflection
}

\author{
Adrian D. C. Chan ${ }^{I}$, Laura Colley ${ }^{I}$, Ryan Dempsey ${ }^{I}$ \\ ${ }^{1}$ Department of Systems and Computer Engineering, Carleton University \\ adrian.chan@carleton.ca
}

\begin{abstract}
Instructors engaging students as partners in curriculum development is a growing pedagogical approach. This can promote a two-way conversation between instructors and students and jointly benefit both sides of the partnership. This paper will take the form of a case study centered on implementation of the Students as Partners Program (SaPP) for two compulsory biomedical engineering courses at Carleton University. Reflections on student partnership and its effects will be considered from multiple perspectives: from the instructor, from the SaPP student, and from a student who took one of the courses. Generalized observations will be presented, as well as reflections that may be applicable to the engineering academic community at large.
\end{abstract}

Keywords: Students as partners, pedagogical partnership, engineering education, case study, reflection.

\section{INTRODUCTION}

Instructors engaging students as partners in curriculum development is a pedagogical approach that has gained momentum over the past decade. The students as partners approach involves the co-creation of curriculum by the instructor and student partner. These partnerships can promote increased student engagement and success, innovations in teaching and learning, and a deeper understanding for both partners of their respective roles within the academic community [1].

This approach has been previously explored in a variety of contexts. In prior exemplars in both a Service-Learning Teaching Assistant program and workshop structure, respectively, faculty appreciated the collegial nature of these student-teacher partnerships and the logistical help the student was able to provide [2], as well as finding value in practical course design feedback [3]. In order to facilitate meaningful dialogue and constructive work, a transition must occur from the traditional hierarchical relationship between the instructor and student, to a partnership where both members are mutually respected and regarded as holders and creators of knowledge [4][5]. These partnerships can provide a space for students where, in contrast to most academic life, they feel their knowledge is unique and valued [6]. Correspondingly, these partnerships can increase student self-esteem and increase equity and inclusion within the classroom [6][7][8][9]. Prior research on the students as partners pedagogical approach has been largely positive for the reasons listed above [10]. There are also criticisms that exist. For example, academic power dynamics may inadvertently suppress authentic communications and actions towards meaningful, positive institutional changes [11]. In addition, accessibility barriers (e.g., physical, technological, and systematic barriers) may exclude the participation of marginalized and underserved groups, including disabled students [11].

This paper presents a case study centered on a students as partners implementation for two biomedical engineering courses at Carleton University. Reflections on the experience and outcomes will be considered from multiple perspectives: from the instructor, from the student partner, and from a student who took one of the courses. Generalized observations will be presented, as well as benefits that may be applicable to the engineering academic community at large. This paper does not aim to argue for or against students as partners; instead, it presents the authors' personal lived experiences and reflections.

\section{BACKGROUND}

\subsection{Description of the Students as Partners Program}

In the Winter 2020 term, Carleton University launched the Students as Partners Program (SaPP) [12]. SaPP offers paid work experience to undergraduate students working with an advisor (i.e., faculty, instructor, contract instructor, educational and instructional developer, or librarian). In the program's first term at the university, a total of $19 \mathrm{SaPP}$ partnerships occurred; Summer and Fall 2020 saw 121 and 137 total partnerships, respectively. The Faculty of Engineering and Design saw 5 partnerships in Winter 2020, 13 in Summer 2020, and 20 in Fall 2020. "The partnership program seeks to engage students in different projects and activities, including, but not limited to: 
- Co-designing and contributing to the development of curriculum;

- Researching, sourcing and organizing course materials, media, and appropriate learning resources;

- Helping design course materials such assessments, cuLearn site, and multimedia tools;

- Formatting and preparing assessments;

- Incorporating educational technologies, whether online or face-to-face;

- Enhancing the use and understanding of assessment criteria;

- Working on a Scholarship of Teaching and Learning (SoTL) project" [12].

SaPP allows students to meaningfully contribute to the academic direction of a project, and requires all the work to be completed before the beginning of the course. Each $\mathrm{SaPP}$ position is funded to a maximum of 130 hours per academic term; all of the funding is provided by Carleton University's Teaching and Learning Services.

\subsection{Description of Courses}

This paper describes two offerings of SaPP, which focused on two different courses: 1) SYSC 3203: Bioelectrical Systems, offered in Fall 2020; and 2) SYSC 4201: Ethics, Research Methods, and Standards for Biomedical Engineers, offered in Winter 2021. The calendar descriptions for these courses are shown in Table 1. Because of the COVID-19 pandemic, all teaching at Carleton University moved online for the 2020/21 academic year, which included these two course offerings. Co-author $\mathrm{AC}$ is the instructor for both of these courses. Co-author LC is the SaPP undergraduate student who worked with AC on both these courses; she had completed both courses in the previous academic year (2019/20), including SYSC 4201 taught by AC in Winter 2020. Coauthor $\mathrm{RD}$ is an undergraduate student who completed SYSC 3203 in Fall 2020. He was approached to contribute to this article after SYSC 3203 was fully completed, and is not enrolled in SYSC 4201 in Winter 2021.

The first SaPP term, which focused on collaborative pedagogical design for SYSC 3203: Bioelectrical Systems, was completed in Summer 2020. This was co-author AC's first time teaching this course. SYSC 3203 is a third-year biomedical engineering course which is compulsory for students in the B.Eng. Biomedical and Electrical Engineering program. It had an enrollment of 32 students for the Fall 2020 term. This course teaches about bioelectrical signals, biomedical sensors, amplifier design, filter design, and biomedical electrical safety. Previous offerings of this course included a course project, where students would design, build, and test a circuit that acquired and processed electromyography (EMG) signals (electrical signals associated with the contraction of skeletal muscles). Essentially, the circuit would acquire
Table 1: Calendar descriptions of courses.

\section{SYSC 3203 Bioelectrical Systems}

Biomedical transducers, sensors, and biomedical actuators. Biomaterials and biocompatibility. Amplifier designs: inverting, noninverting, differential, and bioinstrumentation. Amplifier analysis: gain, sensitivity, distortion and stability. Filter design. Sampling and quantization. Electrical machines. Biomedical electrical safety and standards.

SYSC 4201 Ethics, Research Methods and Standards for Biomedical Engineering

Ethical theories, ethical decision-making, biomedical research ethics: informed consent, confidentiality, privacy, research ethics boards; research methods: hypothesis formulation, data collection, sampling bias, experimental design, statistical literacy; regulations for design, manufacture, certification of medical devices; impact of technology and research (social, political, financial).

EMG signals noninvasively from electrodes on the surface of the skin and use them to trigger a mouse click when the amplitude of the signal passed a threshold; the mouse click could be used as a control input to play a game such as Flappy Bird [13]. The Fall 2020 offering of SYSC 3203 maintained this project; however, it was completed virtually using the circuit simulator LTSpice [14], rather than students prototyping the circuit on a breadboard.

The second SaPP term, which focused on collaborative pedagogical design to adapt SYSC 4201: Ethics, Research Methods, and Standards for Biomedical Engineers for online teaching, was completed in Fall 2020. Unlike SYSC 3203 , this was not the first time AC had taught this course; he had taught this course as an in-person course 10 times previously. SYSC 4201 is a compulsory course for students in the B.Eng. Biomedical and Electrical Engineering and B.Eng. Biomedical and Mechanical Engineering programs, and had an enrollment of 57 students for the Winter 2021 term. This course teaches about ethical theories, biomedical research ethics, research methods, statistical literacy, and medical device regulations. It includes a group research project involving human subjects, which includes a research proposal, research ethics board (REB) application, data collection, data analysis, conference paper, and conference presentation. Because of course time constraints, research themes are chosen to allow for simple and constrained projects to be completed; example themes from previous years include two-point discrimination, heart rate variability, and grip strength using a hand dynamometer. With COVID-19, research themes were chosen to allow data collection to be conducted virtually with ease (e.g., using online tests such as 
https://humanbenchmark.com/). The themes were reaction time, memory, hand-eye coordination, and hearing.

\section{DESCRIPTION OF SAPP COLLABORATIONS}

With COVID-19, the SaPP collaborations were completely online, with weekly scheduled meetings; in fact, AC and LC were working in different countries. Microsoft Teams was used as a collaborative platform, using functionality such as video conferencing (Zoom was primarily used in Summer 2020), chat messaging, cloud storage, and sharing of files, including live cloud editing.

In addition to the course learning objectives, there were underlying educational philosophies that were co-created for the SaPP collaboration:

1. Health and well-being

a. Health and well-being of the students, instructor, and teaching assistants should be an overriding priority.

b. Course workload should be reasonable.

2. Equity, Diversity, and Inclusion (EDI)

a. Course design strives for equity, diversity, and inclusion by having it as a continuous commitment, rather than assuming it is ever fully achieved.

b. Course should have expectations that are clear and communicated early.

c. Students should have appropriate access to any required materials with consideration of potential barriers (e.g., financial, technological).

d. Provide adequate time windows to completed work and limit required, synchronous online activities. This provided a flexible approach for students who have competing demands (e.g., caregiving), who may be in different time zones, and may have unexpected issues (e.g, technology failures, illness).

3. Formative education

a. Students should be given the opportunity to receive feedback and improve during the course. This should be reflected in the evaluation approaches.

b. There should be more frequent, lower stake evaluations. Care should be taken so that these evaluations do not consume students' time (e.g., providing opportunities for assistance, so students are not stuck for extended periods of time).

4. Trust and Respect

a. Give trust and respect to the students from the start (rather than requiring that these be earned over time).

b. Ensure expectations are clear and given in a timely manner, with time flexibility, giving students an opportunity to plan. c. Provide adequate opportunities for students to ask questions and receive assistance.

Pedagogical design decisions for the courses were informed by this educational philosophy, including the choice of using the circuit simulator LTSpice for SYSC 3203 , the use of the online platform (Zoom for SYSC 3203 and Microsoft Teams for SYSC 4201), grading scheme, choice of teaching materials (e.g., choosing required textbooks for SYSC 4201 that were available to students online via the university library).

\section{Multiple Perspective Reflections}

\subsection{Reflections from the Instructor}

The sudden transition to online teaching in the Winter 2020 term due to COVID-19 did not foster the creation of an ideal teaching and learning experience; however, I believe a focus on health and wellness, as well as care and compassion, was helpful for the students in my classes. Having taught more than half the term in-person, I could build upon the existing relationship I had with the students, which was helpful. For the 2020/21 academic year, courses were fully online and it would be the first time that I would be teaching a fully online course. Despite years of teaching experience, this was very daunting for me.

In recent years, I have been increasingly involved with EDI initiatives, with a particular focus on disability and accessibility and women in science and engineering. The transition to online teaching in the Winter 2020 term appeared to have widened the gaps in the educational experiences and opportunities due to a variety of factors, including technological barriers, economic barriers, and increased caregiving responsibilities. In preparation for my 2020/21 teaching, I wanted to take an inclusive design approach, designing at the margins to produce an outcome that is more resilient, adaptable, and inclusive [15]. Inclusive design can create environments that are necessary for some but beneficial for many. I eagerly sought out the SaPP opportunity, as it provided a way of directly including the student voice into my pedagogical design process.

One of the first things I did in my SaPP collaboration with Laura was sharing a draft of my educational philosophy, which we collaboratively refined and shared with students in the course outlines. Beginning with the educational philosophy was helpful because it facilitated a common understanding of why we were making design decisions (e.g., asynchronous versus synchronous lectures, assessment activities, grading scheme). Having a student voice provided perspectives that I had not considered, helped to check (or reveal) my assumptions, and led to a better understanding of the needs and preferences of students. For example, the SaPP collaboration led to the creation of Test \#0 for my courses, which allowed students to go through the entire mechanics of the online test process (e.g., downloading the test from the learning 
management system or LMS, scanning their answers, converting to PDF, uploading and submitting answers to the LMS). As Test \#0 did not count for any grades, it helped to reduce student anxiety associated with learning a new test procedure during a time-limited activity, and revealed student issues and misunderstandings that were resolved before the first assessment test.

I found it interesting that the two courses ended up being quite different in terms of their delivery models, with SYSC 3203 having a greater focus on asynchronous learning (e.g., pre-recorded videos) and SYSC 4201 having a greater focus on synchronous learning (e.g., small interactive breakout rooms, project-focused problem analysis sessions). Compared to my previous course offerings, I found that the pedagogical design of these courses were more informed by the students' needs and preferences, and course content (e.g., deciding what was more core to the course learning objectives versus more peripheral), rather than my own personal habits and preferences. This is not to say that my own habits and preferences did not greatly influence the course design it would be hard for this not to happen and would also lead to an inauthentic outcome — but rather the addition of another perspective had a profound influence. I had similar experiences when co-teaching, particularly across disciplinary boundaries [16].

From a more basic level, the SaPP collaboration helped to ensure early and regular attention towards course preparations. I am guilty of often procrastinating with course preparation; the demands from my research and administrative activities readily provide excuses for that procrastination. I believe part of reason for my procrastination is due to the struggle I have moving through uncertainty. Having a collaborator to discuss areas of uncertainty was enormously helpful for me to continue to move forward. Even if the discussions did not lead to a resolution, there was a comfort and reassurance received from the conversations that we would figure it out.

The SaPP collaboration gave me a higher appreciation of the student perspective, which has helped prevent issues or address them in a timely fashion. As such, in SYSC 3203 and SYSC 4201 I solicited regular, early feedback in both courses to get input on what was working and what could be improved. For SYSC 3203, I was uncertain about what to do with the scheduled synchronous lecture time, but with the student input was able to move towards live, interactive sessions where we worked through example problems. For SYSC 4201, early feedback revealed issues in how I organized material in the LMS, and that I grossly underestimated the course workload. I was able to make changes in the course that, based on additional student input, appears to have successfully resolved these issues. I imagine that without the student input, the teaching and learning environment could turn negative, fostering negative affect (e.g., mistrust, resentfulness, anger, sadness, and stress). Remaining flexible, adaptable, and open to the voices and perspective of students can have a positive impact on student engagement, the student experience, and my own teaching experience.

\subsection{Reflections from the SaPP Student}

The learnings I gained from my SaPP participation can be broadly categorized under three main themes: 1) instructor partnership and empathy, 2) academic engagement with course material, and 3) consideration of equity and inclusion.

Firstly, my SaPP partnership was helpful for the simple act of partnering with Dr. Chan. One of the most significant takeaways I gained from this experience was an evolved perspective of the student-instructor relationship. Prior to $\mathrm{SaPP}$, the relationships I had with all of my professors were, quite predictably, very hierarchical. There was a perceived power dynamic in favour of the instructor (at least from my perspective), and I always had the sense of being "lower on the totem pole", so to speak. In early March 2020, while I was a student in SYSC 4201 with Dr. Chan as my instructor, I approached him to see what he might know about internship opportunities that summer. I can remember carefully choosing my language and rereading my email, not wanting to come off as demanding and thus compromise any help he might be willing to provide - I was only a lowly student, after all!

I am happy to say that partnering with Dr. Chan has helped to revise these prior conceptions. I greatly appreciated being made an equal in curriculum development, with my thoughts sought out and listened to, and found that this experience bolstered my confidence. I found myself able to give my candid opinion on what I liked and didn't like. I also found that technical help was a two-way street - when I was struggling with MATLAB code for the SYSC 3203 project, for instance, Dr. Chan sent over a script to help. Most of all, I have appreciated the rapport we have built and the mutual regard that enables healthy partnership. This experience has helped me in other ways, too: I have gained increased empathy and understanding for my other instructors, understanding some of their intentions when it comes to course development and a greater understanding of the links between content, assessments, and learning objectives.

Secondly, my SaPP participation has also been helpful from an academic perspective. I found that by returning to course material I had already studied with the intent of codeveloping a curriculum, I reaffirmed my existing knowledge, gained a deeper understanding of material, and thought about new perspectives. Transferring the SYSC 3203 project to LTSpice, for example, brought its own set of restrictions - some of the components previously used in the physical offering of the project weren't available in LTSpice's library, and I had to put thought into the underlying purposes of these components within the overall circuit while searching for suitable alternatives. Giving feedback on course readings for SYSC 4201 
required thought into how much of different ethical theories to cover, where the relevant information was, and where an appropriate cut-off within the text might be that ensured students still gained sufficient knowledge. All of these activities broadened my understanding and made me engage more actively with the course material than I had before. One thinks of Bloom's Taxonomy of Learning, with my activities as a SaPP student moving me several rungs up the ladder from Knowledge, Comprehension, and Application to Analysis, Synthesis, and Evaluation [17]. These activities also improved my ability to communicate ideas to an appropriate audience, which will inevitably be relevant throughout my career.

Finally, I found that my experience as a SaPP participant gave me increased awareness and consideration for the ideals of equity and inclusion. Working alongside Dr. Chan to consider all the factors that might impact a student's experience in the courses - including different time zones, Internet access, mental health, and workload from other courses - was a formative learning experience in how these ideals can be actionably implemented within a university environment. Sometimes this came with uncomfortable compromises: to ensure that discussions within SYSC 4201 could be candid, the decision was made to only host these lectures live and forgo recordings, which would of course be non-ideal for students in very different time zones. Sometimes these ideals do not play out perfectly in practice.

I can safely say that my experience as a SaPP student was enormously helpful. This partnership aided me in learning empathy for professors and engaging in an equal partnership with Dr. Chan, helped my academic engagement and knowledge, and gave me a new perspective on the implementation of ideals such as equity and inclusion. It is my opinion that these benefits could extend to other SaPP students engaged in partnerships with faculty in an engineering context.

\subsection{Reflections from a SYSC 3203 Student}

My experience with the course taken, SYSC3203, was largely positive. I found there was a larger emphasis on student engagement and success than what I've grown accustomed to. Primarily, I found that the structure of the course encouraged my own curiosity and growth with the content. The format of the course deliverables facilitated a very natural progression through the learning cycle and the open communication style allowed the instructor to adapt to students' needs throughout the term, as well as keeping student stress manageable. Additionally, the selection of course materials and organization all allowed an appropriate level of accessibility. The natural progression of the content can mainly be attributed to the material found within the course deliverables.

The course had two major sources of grades (among others) throughout the term: bi-weekly tests, and weekly laboratories. The laboratories culminated in a large circuit designed and simulated by the students to measure and process EMG signals from a bicep. The tests and labs focused on real-life applications of the knowledge and skills gained throughout the course.

A typical learning "unit" would develop over a week, where I would:

1. Read the suggested textbook material to gain an overview of the general background

2. Watch the asynchronous lecture videos on the various sub-topics

3. Attempt the 'Check Your Knowledge' section (relevant practice questions); upon any difficulties I would revisit the steps above

4. Make a preliminary attempt at the week's lab, using the skills obtained above

5. Attend the synchronous lecture sessions for extra tips for labs and problem-solving

6. Write the test (every other week)

7. Attend the lab demonstration

8. Write the lab write-up

I found this to be an effective path to success for a number of reasons. This organization was what made the most sense to me given the ordering of the materials on the course page, the progression of the content, and scheduling of test and lab submissions. The difficulty of the content seemed to complement this method, as the textbook would provide a good overview of the theory (as well as some examples). The asynchronous content that followed this was palatably divided into sets of shorter $(\sim 5-10$ minutes $)$ videos that concisely highlighted the relevant theory necessary for problem-solving; this minimized "Zoom fatigue" and kept me engaged. The Check Your Knowledge section provided a guided opportunity to attempt to put the new knowledge to real use. Two to three questions would be posted per unit (mainly design, but some relevant theory on occasion). Since these would later be taken up (in video and/or during synchronous sessions), this was a sort of "training wheels" run at solving the newly learned types of problems. Following this, I felt confident enough to attempt the weekly labs ("ride the bike" myself, if you will). Since the labs built to an overall EMG system, they required genuine design decisions that an engineer might make. They were new problems that were openended enough to make me deeply reflect on the design requirements in order to make those decisions with my lab partner, but directed enough to get us going. The synchronous sessions (twice per week) offered an opportunity to discuss difficulties and get any necessary help from Dr. Chan, including with the week's lab, Check Your Knowledge, etc. They were a more hands-on experience than the asynchronous lectures, often providing further practical examples. The tests would evaluate the problem-solving skills acquired throughout the previous two weeks. I found them to be similar to the labs in their real-world application and design freedom, though slightly more straightforward - they seemed to be an appropriate 
gauge on our grasp of the material. I should also mention the final exam was very similar in format to the tests, except that it was cumulative and longer. The lab demo was when my partner and I showed the teaching assistant our design for the week. He assessed it, and individually asked us pertinent questions about the design and how it worked. The lab write-up was a collaborative effort, again to demonstrate our understanding of the circuit by answering questions about the design. I was satisfied with the course content, as I found the progression described above to be conducive to success in the course. As each piece built upon the previous, my comfort level with the week's material incrementally increased. I did run into problems, of course; however, my stress was mitigated by the open communication with the instructor, among other things.

Each synchronous lecture, the instructor held an anonymous survey asking how caught up students were with the material, on a visual scale. He used this to help tailor his lectures and optimize student engagement. Additionally, Dr. Chan allowed students to use the "raise hand" feature, use the chat feature, or unmute themselves briefly to ask questions freely. This was helpful because it allowed several methods to communicate our concerns, leaving us free to use any combination of these methods that make us the most comfortable. After answering a question (typically complemented by a short example and/or counter-example), Dr. Chan would verify with the student who asked the question whether the explanation was sufficient, and would answer any further questions. I believe this stems from holding the main lectures asynchronously, which allowed more time for a dialogue in the synchronous component.

Apart from lectures, the instructor was keen on getting student feedback. Several times throughout the semester, he provided anonymous surveys with directed prompts on our opinions about course components, such as test length or lab prompts. I appreciated this because I often hold back my opinion because I assume university courses are a rigid plan. In this case it was clear to me that Dr. Chan was on the students' side, willing to accommodate us however necessary in order for us to gain something from the course. It's also worth noting that the instructor made use of this feedback. For example, one of the tests had the majority of students commenting on the difficulty, so Dr. Chan took note of this and made the next test more straightforward. It felt great to have our difficulties acknowledged by the instructor, and contrary to some other courses, I was motivated to work hard. I was encouraged knowing that the instructor could be accommodating.

One last note to the instructor's communication style is that he was readily available by email for several hours of the day. Frequently I found when I sent inquiries to him, even if he was in and out of meetings, he would take the time to promptly respond with detailed explanations. The same goes for the course's teaching assistants as well. I felt encouraged to ask for help, and felt that the students' questions were important to all of the teaching staff. What complemented this strong communication were several accessibility decisions detailed below, which minimized my stress throughout the semester.

One factor in the accessibility of the course is the required materials. In this case, the textbook was The Art of Electronics [18]. This book was very practical in nature, and I suspect I will use it in the future (whether that be academically or otherwise) because of its general electronics design applications. I appreciated this, considering sometimes textbooks I purchase are no longer of use after the course ends. Additionally, I found it very easy to parse because of its down-to-earth writing style. Another required material for the course was the circuit simulator, since the semester was completed online. The program chosen was LTSpice. There are a number of different SPICE (Simulation Program with Integrated Circuit Emphasis) programs available, but few have the benefit of being free. As a student I was happy to save my money, and I think it's fair that the students weren't given an added expense because of the pandemic. The price tag didn't inhibit any features either. All of the circuits still required the same deliberate design choices, and I still got the chance to learn about the ramifications of those choices. The learning was actually made easier, in some sense, than on campus, because it was easy to quickly swap out certain circuit components and investigate the changes. Of course, physically assembling the circuits would be nice, but the advantages to simulation are nonetheless present. It was also a great tool to demonstrate the effects of any circuit, regardless of whether this was for a lab or for some other portion of the course - Dr. Chan used it in lectures to complement his teaching, I used it a lot while reading the textbook, and honestly I plan to use it in the future too. Dr. Chan also provided enough material on LTSpice to make getting started with it seamless. I personally had never used any SPICE software going in and found myself very comfortable with the software early on. One particular part that helped was Lab 0. Lab 0 was worth no grades, and was just a trivial circuit for the purpose of familiarizing us with LTSpice as well as the lab submission process. I found it helpful because it was a nice dry run for the first graded lab, which helped familiarize myself with the software and submission process, but also gave me peace of mind that I could tackle Lab 1 without administrative difficulties.

A similar process was used for tests, wherein Test 0 , a trivial circuit question worth 0 marks, was written to familiarize us with the test-taking process. This was also beneficial for my peace of mind, though there was no software to acquaint myself with, as in Lab 0. On the topic of tests, I appreciated the way they were handled. Each test had 24 hours in which you could start writing the test. Once you start writing the test, the timer began. This was helpful because I had control over what part of my day I could write the test. Additionally, I have a friend living across the world who greatly appreciated not needing to write the 
tests in the middle of the night! Each test was 30 minutes long but we were given 1.5 hours, with the additional hour to account for associated logistics (e.g., downloading test, and submitting answers). We signed an honour statement indicating we would not use the Internet nor go over the time limit. This reduced my stress with scanning and uploading my responses, knowing I had such a large time buffer. I actually made good use of this extra time once, debugging my malfunctioning scanner. If it weren't for that buffer I would have definitely been more anxious which would have inhibited my ability to perform. I find in this pandemic many instructors err on the side of not letting students cheat by employing strategies such as sequential exams in which you can't consult previous questions and/or strict automated proctoring software, to name a few. However, in this case I think it's clear that the instructor erred on the side of compassion. Under this format, students were not stressed as a result of the adaptations to the pandemic. Instead, we were trusted, as adults, to uphold academic integrity. This is something I really appreciated, and it motivated me to work hard.

Another component of note was the compassionate grading scheme. Firstly, there were two grading scheme options: one that used all five tests, and one that dropped the lowest test and added that weight to the final exam. Whichever scheme yielded the higher final grade was implemented. This choice further solidified my comfort because I felt that my final grade would truly be a representation of my level of effort, while allowing me to make some mistakes (as humans do). Another note about the grading scheme is that $5 \%$ of the final grade was allocated for reflection on the course material. There was $1 \%$ for completing one midterm and one final evaluation on the experience with one's lab partner. The remaining $4 \%$ were for completing at least 10 of 12 weekly reflections. These weekly reflections required posting on the cuLearn Weekly Reflections forum the answers to the following prompts:

1. What is something new I learned this week or found interesting?

2. What is something I would like to learn more about or am having difficulties with?

The last week's questions were altered, being a reflection on the semester as a whole. I appreciated these choices because they motivated me to reflect on the significance of what I learned throughout the term. Sometimes we can become a bit mechanical in the learning process and lose focus of the "why", especially in formal education. The weekly reflections remedied this by making me consider the impact the course was having on me, as well as the next steps for the next week. The lab evaluations were also helpful to figure out what was working and what wasn't working for myself and my lab partner.

My experience with SYSC3203 this fall was truly my favourite course experience of my undergraduate degree thus far. Of course it helped that I enjoyed the content, but the way the course was run was crucial. The learning materials facilitated a very smooth progression through the learning cycle, the open communication allowed me to voice my issues and have them addressed, and there were several accessibility decisions that minimized my stress. Throughout the course I truly resonated with the learning experience and I hope that future courses will implement similar design choices.

\section{DISCUSSION}

This paper provides multiple perspectives of a shared SaPP experience, including the perspectives of the instructor, the SaPP student, and a student who was enrolled in one of the courses. The reflections appear to demonstrate a strong coherency between the course design and student experience; that is, the intent of the instructor and SaPP student in their pedagogical design were reflected in the experience of the student in the course.

Open coding of the three written reflections was performed in Miro [19]. The codes were then grouped into categories and themes. This qualitative analysis yielded three themes. The first theme was pedagogical design. There was a student-centered approach, which included student feedback and an openness to being flexible and adaptable to student needs and preferences. The student in the course found the pedagogical design to be an organized and effective learning experience. The second theme was the student-instructor relationship. Several important characteristics to this relationship emerged, including open communications, trust, empathy, and compassion for one another. The third theme was accessibility. An EDI approach was taken for course design decisions, which helped improve the accessibility of the course and support student mental health and engagement.

\section{CONCLUSIONS}

Through the SaPP pedagogical approach, an effective, accessible, and resilient teaching and learning experience was able to be constructed. Reflections from the instructor, the SaPP student, and a student taking the course demonstrated these outcomes. In addition, both the instructor and SaPP student learned and grew from the SaPP collaboration: acquiring new knowledge and skills, evolving attitudes, and challenging stereotypes and biases.

\section{Acknowledgements}

The authors would like to thank Carleton University's Teaching and Learning Services for their funding of the Students as Partners Program.

\section{References}

[1] Alison Cook-Sather, Catherine Bovill, and Peter Felten, "Outcomes of Student-Faculty Partnerships", in 
Engaging Students As Partners in Learning and Teaching: A Guide for Faculty. Hoboken, NJ: Wiley, 2014, 304 pp. \{ISBN: 978-1-118-43458-1\}

[2] Gail S. Begley, Becca Berkey, Lisa Roe, and Hilary E. Y. Schuldt, "Becoming partners: Faculty come to appreciate undergraduates as teaching partners in a service-learning teaching assistant program," International Journal for Students as Partners, vol. 3, no. 1, pp. 89-105, 2019. Available as of February 27, 2021, from https://mulpress.mcmaster.ca/ijsap/article/view/3669.

[3] Catherine Anne Snelling, Beth R. Loveys, Sophie Karanicolas, Nathan James Schofield, William Carlson-Jones, Joanne Weissgerber, Ruby Edmonds, Jenny Ngu, "Partnership through co-creation: Lessons learnt at the University of Adelaide," International Journal for Students as Partners, vol. 3, no. 2, pp. 6277, 2019. Available as of February 27, 2021, from https://mulpress.mcmaster.ca/ijsap/article/view/3799.

[4] Gabrielle Foran, Kris Knorr, and Rebecca L Taylor, "Evaluating factors contributing to positive partnership work in a students-as-consultants partnership program," International Journal for Students as Partners, vol. 4, no. 2, pp. 27-44, 2020. Available as of February 27, 2021, from https://mulpress.mcmaster.ca/ijsap/article/view/4095.

[5] Anita Ntem and Alison Cook-Sather, "Resistances and resiliencies in student-faculty pedagogical partnership," International Journal for Students as Partners, vol. 2, no. 1, pp. 82-96, 2018. Available as of February 27, 2021, from https://mulpress.mcmaster.ca/ijsap/article/view/3372.

[6] Alise de Bie, Elizabeth Marquis, Alison Cook-Sather and Leslie Patricia Luqueño, "Valuing Knowledge(s) and Cultivating Confidence: Contributions of StudentFaculty Pedagogical Partnerships to Epistemic Justice", in Strategies for Fostering Inclusive Classrooms in Higher Education: International Perspectives on Equity and Inclusion. Bingley, UK: Emerald Group Publishing, 2019, 308 pp. \{ISBN: 978-1-787-56061-1\}

[7] Alison Cook-Sather, "Respecting voices: how the cocreation of teaching and learning can support academic staff, underrepresented students, and equitable practices," Higher Education, vol. 79, pp. 885-901, 2020. Available as of February 27, 2021, from https://link.springer.com/article/10.1007/s10734-01900445-w.

[8] Alison Cook-Sather, Srikripa Krishna Prasad, Elizabeth Marquis, and Anita Ntem, "Mobilizing a Culture Shift on Campus: Underrepresented Students as Educational Developers," New Directions for Teaching \& Learning, vol. 2019, no. 159 , pp. 21-30, 2020. Available as of February 27, 2021, from https://onlinelibrary.wiley.com/doi/abs/10.1002/t1.203 45.

[9] Kate Brown, Alise de Bie, Akshay Aggarwal, Ryan Joslin, Sarah Williams-Habibi, and Vipusaayini
Sivanesanathan. "Students with disabilities as partners: A case study on user testing an accessibility website", International Journal for Students as Partners, vol. 4, no. 2, pp. 97-109, 2020. Available as of February 27, 2019, from https://mulpress.mcmaster.ca/ijsap/article/view/4051.

[10] Lucy Mercer-Mapstone, Sam Lucie Dvorakova, Kelly E. Matthews, Sophia Abbot, Breagh Cheng, Peter Felten, Kris Knorr, Elizabeth Marquis, Rafaella Shammas, Kelly Swaim, "A systematic literature review of students as partners in higher education," International Journal for Students as Partners, vol. 1, no. 1, pp. 1-23, 2017. Available as of February 27, 2021, from https://mulpress.mcmaster.ca/ijsap/article/view/3119.

[11] Alise de Bie, "Respectfully distrusting 'Students as Partners' practice in higher education: applying a Mad politics of partnership", Teaching in Higher Education, 2020. Available as of February 27, 2021, from https://www.tandfonline.com/doi/abs/10.1080/135625 17.2020.1736023? journalCode $=$ cthe 20 .

[12] "Students as Partners Program (SaPP)." Carleton University. Available as of February 12, 2021, at https://carleton.ca/discoverycentre/fundingopportunities/students-as-partners-program-sapp/.

[13] "Play Flappy Bird.” Available as of March 2, 2021, from https://flappybird.io/.

[14] "LTspice." Analog.com. Available as of March 2, 2021, from https://www.analog.com/en/designcenter/design-tools-and-calculators/ltspicesimulator.html\#.

[15] Jutta Treviranus, "Inclusive Design: The Bell Curve, the Starburst and the Virtuous Tornado", Medium.com. Available as of February 24, 2021, from https://medium.com/@jutta.trevira/inclusive-designthe-bell-curve-the-starburst-and-the-virtuous-tornado6094f797b1bf.

[16] Beth Robertson and Adrian Chan, "A multidisciplinary learning experience for education in accessibility", in Proc. CEEA Canadian Engineering Education Conf., CEEC19, (Ottawa, ON: 8-12 June 2011), 6 pp., 2019.

[17] Nancy E. Adams, "Bloom's taxonomy of cognitive learning objectives," Journal of the Medical Library Association, vol. 103, no. 3, pp. 152-153, 2015. Available as of February 27, 2021, from https://www.ncbi.nlm.nih.gov/pmc/articles/PMC4511 $\underline{057 /}$.

[18] Paul Horowitz and Winfield Hill, The Art of Electronics. Cambridge, MA: Cambridge University Press, 2015 ( $3^{\text {rd }}$ ed.), 1227 pp. \{ISBN: 978-0-52180926-9\}

[19] "Miro". Available as of May 2nd 2021, from https://miro.com/app/dashboard/. 